J. Lake Sci. (湖泊科学), 2011, 23(1): 1-8

http: //www.jlakes.org. E-mail : jlakes@niglas.ac.cn

(C) 2011 by Journal of Lake Sciences

\title{
溶解性有机质与水生生物的直接相互作用研究进展
}

\author{
陈 蕾, 沈超峰, 陈英旭 \\ (浙江大学环境与资源学院环境保护研究所,杭州 310029)
}

\begin{abstract}
摘 要: 溶解性有机质广泛存在于天然水体中, 具有重要的生态与环境意义. 然而在环境科学领域内, 天然水体中的溶解 性有机质长期以来仅仅被作为惰性的吸附剂对待, 其自身的与生物的直接作用却一直被忽视. 近年来, 越来越多的研究 证据表明溶解性有机质自身即具有生物效应. 它能在生物表面吸附, 并影响细胞膜的电化学性质与膜渗透性, 能被生物 吸收, 进而诱导生物体内化学物质应激反应, 诱导并调节生物体内代谢酶的活性, 同时对生物产生一定的内分泌干扰效 应. 溶解性有机质对生物的轻度刺激作用是影响污染物的生物浓缩及毒性效应的重要因子之一, 系统地研究其中的机制 对评价实际环境中污染物的生态风险有着重要意义. 本文综述了溶解性有机质与生物的直接作用的相关研究成果, 并探 讨目前此分支领域研究中出现的瓶颈问题.
\end{abstract}

关键词: 溶解性有机质; 水生生物; 生物效应; 轻度刺激

\section{Direct interaction of dissolved organic matter with aquatic organisms}

\author{
CHEN Lei, SHEN Chaofeng \& CHEN Yingxu \\ (Institute of Environmental Science and Technology, Zhejiang University, Hangzhou 310029, P. R. China)
}

\begin{abstract}
Dissolved organic matter is widely present in aquatic environment with great ecological and environmental significance. In freshwater ecosystems, dissolved organic matter exceeds all living organisms by more than one order of magnitude. The ability of dissolved organic matter to affect aquatic biota indirectly by exerting strong control on the surrounding biogeochemical conditions is well established due to their ability to complex inorganic cations, including metals and a variety of organic compounds. In contrast, the potential direct interaction of these amphiphilic compounds with aquatic organisms themselves has been largely overlooked until recently. However, in the past decade, there has been growing recognition that dissolved organic matter can directly affect the physiology of organisms. Because of the chemical building blocks and physicochemical behavior of dissolved organic matter, researchers have postulated that these substances have a xenobiotic character, with effects such as sorption of dissolved organic matter on living membranes, alterations in membrane electrophysiological characteristics and permeability, induction of chemical stress defense reactions, induction and modulation of biotransformation enzymes and displaying hormone-like effects and so on. These interactions will impact the bioconcentration and toxic effects of environmental contaminants. Making these mechanisms clear is of critical importance in ecological understanding and risk assessment of contaminants. From the view of ecology, these interactions are thought to be a means of lifespan expansion; however, given the existence of multi-contaminants, these interactions will impact the bioconcentration and toxic effects of environmental contaminants. However, the complexity of dissolved organic matter and the variety of the methods by which the dissolved organic matter is obtained have made the explanation and the comparison between labs rather difficult. The gap between the lab and the field research also needs to be filled. In this review, the research progress on direct interaction of dissolved organic matter with organisms was introduced in detail and the bottleneck of this research aspect was discussed as well.
\end{abstract}

Keywords : Dissolved organic matter; aquatic organisms; bio-effect; mild stress

溶解性有机质 (Dissolved Organic Matter, DOM) 是天然水体中普遍存在的环境物质,其与环境污染物的 相互作用、对污染物的环境行为与生物毒性的影响早已引起人们的关注. 已有的研究中, 溶解性有机质一直

* 2010-02-15 收稿;2010-03-22 收修改稿. 陈蕾,女,1985 年生, 博士研究生; E-mail: clymcl@ zju. edu. cn. 
被视为惰性的环境因子或天然的吸附剂, 探讨其对污染物生物效应的影响几乎都是从溶解性有机质与污染 物的结合物体积大小的角度进行, 然而却不能解释所有的实验结果. 近些年来, 越来越多的研究表明溶解性 有机质自身即能对生物产生不利影响. 这些研究结果将使环境科研工作者重新审视溶解性有机质的环境意 义,尤其是溶解性有机质可以使污染物对水生生物的影响发生改变,值得研究者的重视. 本文对溶解性有机 质与水生生物的相互作用作了系统的文献调研, 对已发现的溶解性有机质的直接生物效应可能导致污染物 对水生生物的影响发生的改变进行了论述, 并对目前该研究方向上的瓶颈问题作了深人的探讨. 从溶解性 有机质的直接生物效应这一常被忽视的角度进行深人的研究, 对充分解释溶解性有机质对污染物生物效应 的影响有着重大的作用, 同时也有助于我们更加准确地评价污染物进人实际环境后的生态风险. 因此, 溶解 性有机质的直接生物效应将会成为未来污染物生态风险研究中考虑的重要因素之一.

\section{1 溶解性有机质}

\section{1 溶解性有机质的特点}

溶解性有机质来源于动植物体的分解残体, 结构和组分十分复杂, 且物理上极不均匀的有机混合物. 它 既含有游离的氨基酸、糖类等低分子量物质, 又含有酶、氨基糖、多酚和腐殖酸等多种大分子成分 ${ }^{[1]}$. 在结构 上,水体中的溶解性有机质具有苯环羧基、酚基官能团构成的聚苯环和某些基于氮和硫原子的基团或键,所 含官能团主要有羧基、醇羟基、酚羟基、醌型羰基和酮型羰基等,其中羧基约占总酸性基团的 $60 \%-90 \%{ }^{[2]}$. 因溶解性有机质结构与成分的高度复杂性, 目前仍没有方法清晰描述其结构. 腐殖质是溶解性有机质的主 要成分, 占溶解性有机质总有机碳含量的 $60 \%-80 \%$, 包括腐殖酸、富里酸等. 腐殖质是动植物残体在水体、 土壤中经过长期的物理、化学、生物作用等过程转化而成的一类高分子物质. 水体中的腐殖质源自植物和土 壤的陆生来源 (外来物质) 以及水生体系自身生物活动产生的物质 (自生物质) ${ }^{[3-4]}$.

目前溶解性有机质仅有操作上的定义, 即溶液中能通过 $0.46 \mu \mathrm{m}$ 孔径的有机组分 ${ }^{[5]}$. 在相关研究中通常 将其分为亲水性组分与疏水性组分, 芳香碳含量、 $\mathrm{H} / \mathrm{C}$ 原子数比、 $\mathrm{O} / \mathrm{C}$ 原子数比、摩尔吸收系数及分子量等 也是常用的表征参数. 水中溶解性有机质的浓度与结构具有显著的地域差异性. 天然水体中溶解性有机质 的浓度范围为 $1-40 \mathrm{mg} / \mathrm{L}$, 海水中平均为 $0.5 \mathrm{mg} / \mathrm{L}$, 江河中平均可达 $7 \mathrm{mg} / \mathrm{L}^{[2]}$.

\section{2 溶解性有机质的生态与环境意义}

作为全球碳循环的重要组成部分, 溶解性有机质对全球变化有重要影响. 它也是生态系统中能量与物 质循环的重要途径, 与生态系统的各个重要环节密切相关; 在流域与湖泊水生生态系统中, 天然有机质在各 种物理、化学和生物过程中均产生重要影响, 是各种养分 (碳、氮、磷和硅) 循环的关键环节, 是异养型微生物 所需能源的主要提供者, 是各种水体物理化学条件 (溶解氧、酸碱度、颜色)、光合作用和二氧化碳水气交换

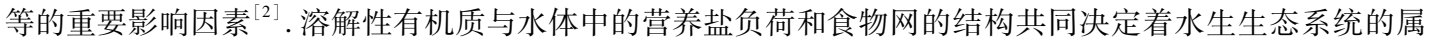
性和动态, 它与养分 (氮和磷) 之间的耦合关系维持着整个水生生态系统的结构与功能 ${ }^{[6]}$.

溶解性有机质在化学结构上既具有羧基、酚基等亲水基团, 又具有脂肪碳链、芳环等疏水基团, 因而溶 解性有机质是天然水体环境有毒污染物的重要化学络合剂和吸附剂, 直接影响它们的迁移转化、毒性、生物 地球化学循环及归宿 ${ }^{[7-10]}$. 溶解性有机质能与金属离子形成有机金属络合物, 导致金属离子生物地球化学行 为的改变, 影响其溶解性、生物有效性、与微粒之间的相互作用, 并改变它们的毒性. 吸附作用是溶解性有机 质控制环境中有机污染物行为与毒性的主要方式,通过建立模型预测发现,溶解性有机质主要 $(33 \%-86 \%)$ 控制着有机污染物在水体环境中的迁移转化行为和归宿 ${ }^{[11]}$. 因此, 溶解性有机质是重要的环境因子, 特别是 在环境污染物的风险评价中有着举足轻重的作用.

众多研究表明, 溶解性有机质存在时生物对污染物的浓缩系数会显著降低 ${ }^{[12]}$. 对此公认的解释是溶解 性有机质与疏水性有机污染物结合以后, 形成的结合物由于分子太大、极性太强而不能穿透生物膜而具有 极低的生物有效性 ${ }^{[13]}$.

然而也有一些研究得到了相反的结论 ${ }^{[12,14-16]}$. Qiao 等 ${ }^{[16]}$ 研究了溶解性有机质存在时虹䲡鱼对数种多氯联 苯同系物的累积情况, 得出低浓度腐殖酸 $(1.5 \mathrm{mg} / \mathrm{L})$ 对 $\lg k_{\mathrm{ow}}$ 在 4-5 范围内的有机物的生物浓缩系数具有显著 提高作用的结论. Chen 等 ${ }^{[17]}$ 发现基于自由溶解态浓度的苯并荧葱生物浓度效应曲线随腐殖酸的加人而改变, 
但略偏离纯水中的浓度-效应曲线 $95 \%$ 置信区间. 由此他们推测一部分的苯并荧蒽与腐殖酸结合物亦具有生 物有效性与毒性. 溶解性有机质的来源种类、含量及有机化合物的结构是影响实验结果的可能因素 ${ }^{[12,18]}$, 然而 要清楚地解释这些不一致的研究结果,仍需对溶解性有机质影响污染物生物效应的机制进行深人的研究.

\section{2 溶解性有机质与生物的直接相互作用}

在过去绝大多数研究中, 溶解性有机质是被作为惰性的环境因子或天然的吸附剂看待. 然而, 近年来有 研究者提出溶解性有机质具有异生质的特点, 应将其视为多种化学物质的混合体 ${ }^{[19-20]}$. 溶解性有机质能够 穿透生物细胞膜 ${ }^{[21-24]}$, 并影响细胞膜的生化特性与离子流 ${ }^{[25-28]}$; 能够对生物产生化学胁迫而诱发生物体内 的一系列应激反应, 如热休克蛋白 (Hsp) 表达的增加、生物转化酶的表达及对生物外排系统载体的竞 争 ${ }^{[29-32]}$; 而且, 溶解性有机质能表现出一定程度的内分泌干扰效应 ${ }^{[33-37]}$.

\section{1 溶解性有机质在生物表面的吸附}

早在 1985 年, Munster ${ }^{[21]}$ 通过对腐殖质电泳特性的研究, 首次提出腐殖质与生物表面的相互作用; 接着 Visser ${ }^{[22]}$ 也提出腐殖质与表面活性剂类似, 能直接作用于微生物表面, 进而影响其代谢活动. Campbell 等 ${ }^{[38]}$ 通过透射电子显微镜证明溶解性有机质在浮游植物及鱼鳃细胞表面的累积, 并发现溶解性有机质在细胞表 面的吸附作用与环境 $\mathrm{pH}$ 、溶解性有机质的浓度密切相关.

然而溶解性有机质在生物表面的吸附机制尚不明确.一方面有研究发现这种吸附作用在酸性条件下更 显著, 因而有学者提出了溶解性有机质的负电荷基团与生物表面以氢键相结合的作用机制 ${ }^{[38]}$; 另一方面也 有研究指出富里酸中疏水性更强的组分与生物的吸附作用更强, 溶解性有机质与生物表面的疏水作用机制 由此被提出 ${ }^{[23]}$. Elayan 等 ${ }^{[24]}$ 从分子水平对吸附机制进行了研究. 他们利用 ${ }^{31} \mathrm{P}$ 核磁共振技术研究三种不同 来源的腐殖酸对模拟生物细胞膜的影响, 发现三种腐殖酸均对模拟生物膜的双分子层产生了扰动, 据此他 们提出吸附/吸收模型, 即腐殖酸先通过氢键与静电作用吸附到细胞膜上, 之后腐殖酸的疏水组分通过疏水 作用进人双分子层内进而被细胞跨膜吸收.

\section{2 溶解性有机质对生物细胞膜的影响}

当受到外界物质的干扰时, 细胞膜是细胞最先感应到、也是最敏感的部位. 溶解性有机质在生物表面的 吸附作用具有普遍性, 而且溶解性有机质具有类似表面活性剂的双极性基团, 当其粘附于细胞表面时, 细胞 膜的相关性质将受到影响. Vigneault 等 ${ }^{[25]}$ 的研究指出天然水体中的腐殖质能增强浮游植物细胞膜及模型细 胞膜对亲脂性物质的渗透性. 溶解性有机质影响钠、钾离子代谢的研究已有报道 ${ }^{[26,28]}$. 如 Glover 等 ${ }^{[26]}$ 发现 腐殖质能显著提高大型蚤细胞钠离子的跨膜运输. 这是钠离子流的一种非竞争性刺激效应, 而腐殖质与膜 的结合导致细胞膜的通透性发生了改变是极可能的一种机制. 细胞膜电化学性质的改变经常用来指示外来 物质对细胞的不利影响 ${ }^{[39]}$. Manusadzianas 等 ${ }^{[27]}$ 发现溶解性有机质对细胞膜静息电位产生影响, 细胞膜的去 极化作用与溶解性有机质的浓度显著相关. Galvez 等 ${ }^{[28]}$ 通过体内与体外试验观察到溶解性有机质存在时鱼 鳃上皮细胞跨上皮细胞电位 (Transepithelial Potential, TEP) 的超极化, 其程度与溶解性有机质的芳香度相 关. 同时他们也推断溶解性有机质使细胞膜电化学性质发生改变是其导致细胞膜渗透性与离子运输性能发 生改变的可能机制. 此外, 溶解性有机质对细胞膜的化学干扰亦被提出, 主要体现为细胞膜的脂质过氧化, 如片脚类生物 ${ }^{[0041]}$. 其中的机制很可能是进人细胞内的小分子腐殖质诱导胞内活性氧物质的生成, 进而引 起细胞膜的脂质过氧化.

\section{3 溶解性有机质对生物防御系统的影响}

天然有机质能否被生物吸收一直是倍受争议的话题. 近年来越来越多的证据表明腐殖质能够被生物体 吸收, 特别是分子量小于 $3.5 \mathrm{kDa}$ 的组分极易通过生物细胞膜 ${ }^{[6]}$. 研究指出水溶性、离子性的腐殖质通常分 子量很小 $\left(0.5 \mathrm{kDa}\right.$ 左右), 而且结构规整, 在天然水体中通常以多价氧离子键、酯键或氢键桥联存在 ${ }^{[42-43]}$. 虽 然这些小分子的腐殖质占溶解性有机质的比例很小, 但其能通过细胞膜进人细胞内部, 足以对细胞的生理 活动产生影响 ${ }^{[9]}$.

进人细胞内的物质可能会激活细胞内的防御系统, 应激蛋白即热休克蛋白 (Hsp) 是一类非特异性的细 胞保护蛋白, 是细胞受到外界压力时极敏感的生物标志物. 即使在较低浓度的腐殖酸的暴露下, 生物体内 
Hsp-70 的浓度也会有显著的增加 ${ }^{[44-45]}$.

生物体能通过代谢清除进人体内的外来物质. 研究表明生物对溶解性有机质的暴露能诱导代谢酶的表 达 $^{[29,44-45]}$. Menzel 等 ${ }^{[30]}$ 在线虫对腐殖酸暴露的研究中发现线虫体内谷胱甘肽硫转移酶、抗氧化物酶的含量 很高, 同时也从分子水平上发现腐殖酸暴露下相关基因的上调. 活体与离体试验结果指出由 $\mathrm{Ah}$ 受体依赖的 细胞色素 P450 酶能被腐殖质诱导和调节 ${ }^{[31,46]}$. 进一步将溶解性有机质进行光降解后发现溶解性有机质中 光稳定性与光不稳定的成分均可能是 $A h$ 受体效应诱导因子 ${ }^{[47]}$.

生物体具备一种异生质外排机制以应对外来有害物质, 它是细胞膜上结合的一种含磷糖蛋白将进人细 胞内的有毒物质或细胞内的代谢物泵出细胞外的过程 ${ }^{[27]}$. 溶解性有机质类似于异生质, 它的广泛存在将与 进人细胞内的其它毒物竞争泵蛋白, 而影响其它环境毒物的正常排出. Timofeyev 等 ${ }^{[32]}$ 对片脚类生物进行溶 解性有机质的暴露, 以细胞内菼光染料 (rhodamine B) 的消失表征生物外排异生质的能力. 研究结果发现, 与 对照相比, 经腐殖质暴露的受试生物体内细胞中苂光染料的残留量显著增加, 说明溶解性有机质确实能影 响生物排除其它毒物的能力.

\section{4 溶解性有机质对生物内分泌系统的干扰}

早在 2001 年 Hoss 等 ${ }^{[33]}$ 就发现富里酸对线虫的繁殖能够产生影响. 他们将线虫暴露于天然水体中提取 分离的富里酸, 发现低浓度的富里酸促进其繁殖, 而高浓度 $(>5 \mathrm{mg} / \mathrm{L})$ 时显著抑制其繁殖能力, 而天然水体 中的溶解性有机质绝大多数是在此高浓度范围内 ${ }^{[12]}$. 通过建立模型可以估算溶解性有机质潜在的内分泌干 扰效应这一因子与溶解性有机质作为能源物质促生物生长这一因子分别对溶解性有机质暴露下生物繁殖 能力变化的贡献. 结果发现, 在接近真实环境浓度的溶解性有机质的暴露下, 溶解性有机质的内分泌干扰作 用占主导地位 ${ }^{[34]}$.

溶解性有机质对水生生物性别分化的影响也引起了部分研究者的关注. 由于天然河水中提取的溶解性有 机质可能会受到其它化学物质或植物激素的污染, 为避免这一因素对实验结果产生的干扰, 一些研究者采用 人工合成的腐殖质代替物 HS1500 进行实验. HS1500 是一种由多酚类物质经自然氧化而形成的平均分子量在 $1.5 \mathrm{kDa}$ 的产物 ${ }^{[35]}$. Meinelt 等 ${ }^{[35]}$ 的研究发现, 剑尾鱼和非洲爪蟾随着暴露 HS1500 浓度的增加而一致地出现雌 性化现象, 在较高浓度时雌雄的主导地位发生了转变. 可见, 溶解性有机质存在一定的内分泌干扰风险.

溶解性有机质产生内分泌干扰效应的机制尚未清楚, 但已有一些研究者从分子学角度进行探索. Lutz 等 ${ }^{[36]}$ 将爪蟾对 HS1500 进行活体暴露, 然后通过菼光定量 PCR 技术 (RT-PCR) 检测其体内雌激素受体 mRNA(ER-mRNA) 的含量, 发现 ER-mRNA 的水平显著高于对照, 说明溶解性有机质是通过雌激素受体的途径 对生物性别分化产生影响. 同时, Lutz 等 ${ }^{[36]}$ 对腐殖酸引起甲状腺疾病 ${ }^{[48]}$ 的机制进行了研究, 通过 RT-PCR 技 术发现与抗甲状腺激素相关的基因 TSH $\beta$-mRNA 的表达水平得到了提高. 然而, 在 Janosek 等 ${ }^{[49]}$ 对重组了雌 激素受体基因的人体与小鼠细胞进行的体外试验研究中, 天然腐殖质提取物的雌激素效应并未发现, 相反 其表现出了一定的抗䧳激素效应, 这表明溶解性有机质对生物内分泌系统干扰的途径并不仅仅是雌激素受 体途径. 溶解性有机质结构中产生内分泌干扰效应的基团尚未确定, 有研究者提出烷基酚和间苯二酚的结 构可能是起作用的基团 ${ }^{[37]}$,仍待进一步的证实.

\section{5 溶解性有机质与生物的直接作用对污染物生物效应的影响}

溶解性有机质易粘附于生物表面的性质将加速水体中与溶解性有机质结合的污染物向生物表面的迁 移, 尤其对疏水性有机污染物 ${ }^{[50]}$. 腐殖质的存在也能增加重金属离子在细胞表面的吸附位点数 ${ }^{[51]}$. 对某些 重金属如铅, 重金属一腐殖质结合物能与细胞膜的吸附、吸收位点形成三重结合体, 这在重金属的生物吸收 过程中起着重要的作用, 例如有研究发现重金属在藻类细胞壁内的主要形态为重金属一腐殖质结合态 ${ }^{[52-53]}$.

溶解性有机质对生物细胞膜的电化学性质、流动性以及离子通道的影响, 势必影响细胞膜对污染物的 跨膜吸收过程. 溶解性有机质对生物产生胁迫效应, 激活生物自身的应激解毒系统, 从生态学角度这种轻度 刺激作用可能对生物应对外界不利环境、增长寿命有着重要的作用 ${ }^{[20,37,54]}$. 然而从另一角度, 当水体环境中 存在多种污染物, 溶解性有机质诱导的应激作用可能会加强生物抵抗外来有害物质的能力, 也可能会因为 溶解性有机质对外排及代谢功能的竞争作用而弱化生物抵抗污染物不利影响的能力.

溶解性有机质影响污染物的生物效应的研究中出现的相悖的结论, 其机制尚不清楚, 也许溶解性有机 
质和生物间的直接作用是这些表观现象不一致的可能原因,然而这方面的研究才刚刚开始. Wiegand 等 ${ }^{[55]}$ 研究了溶解性有机质存在下夹杂带丝蚓对百草枯的浓缩系数及谷胱甘肽硫转移酶、抗氧化物酶、过氧化物 酶的变化关系, 发现了一些规律. 深人了解溶解性有机质对生物的直接作用在污染物的生物吸收及致毒过 程中担任的角色将有利于我们准确预测污染物的环境风险.

\section{3 溶解性有机质与生物直接相互作用研究的瓶颈}

在文献调研的过程中发现与溶解性有机质对生物的直接生物效应相关的研究为数不多, 甚至溶解性有 机质对生物的不利影响的相关研究在全球仅几个课题组在开展. 然而溶解性有机质自身的生物效应对我们 评价污染物进人真实环境后的生态风险有着重要的意义,应该受到环境科学家的重视. 在文献调研的过程 中作者注意到,在已有的溶解性有机质生物效应的研究工作中的某些问题导致了其进展缓慢.

\section{1 溶解性有机质的复杂性与生物效应的轻微性}

天然环境中的溶解性有机质是具有多重性质、结构高度复杂的混合物 , 由于自然环境合成与分解过程 的多样性,溶解性有机质的组成成分可以认为是 “无穷的”, 完全不可能将之分离鉴定, 因而很多情况下能清 楚地解释某些数据或现象都存在着很大的困难 ${ }^{[56]}$. 由于溶解性有机质的异质性与现有技术的能力有限, 任 一种方法, 无论是单独还是联合使用, 都只能反映溶解性有机质的一个方面. 而且, 溶解性有机质真实的性 质并非各单独面的线性相加的结果. 除此之外, 天然有机质会吸附各种污染物, 导致在分析实验结果时很难 排除其它污染物的影响.

虽然溶解性有机质的生物效应不断被报道, 但其对生物的化学胁迫与一般污染物相比更轻微 ${ }^{[37]}$. 而且 溶解性有机质异于环境污染物, 它存在于生物进化的过程之中, 它的生物效应需要从生态系统的水平来评 价. 正是由于溶解性有机质的复杂性与已报道生物效应的轻微性, 溶解性有机质生物效应未能引起很多研 究者的兴趣.

\section{2 提取方法的多样性}

溶解性有机质结构的复杂性与性质的不可预知性已被大家公认, 因而研究者通常针对自己研究采用最 有利的方法来分离、表征溶解性有机质. 目前文献中已出现近百个有关溶解性有机质的简称或缩写, 恰恰反 映了研究者们分离、表征方法的多样性 ${ }^{[56]}$. 出现过的分类方法有基于不同组分的、基于物理性质的及基于化 学性质的方法等.

基于不同组分的方法较为常见. 经常具有相似大小或溶解度的则被归为一类, 然而这种方法得到的组 分总是复杂的化学混合物,其化学组成往往与使用的具体方法密切相关. 基于物理性质的分类方法首先是 根据能否通过 $0.45 \mu \mathrm{m}$ 或 $0.22 \mu \mathrm{m}$ 滤膜分为溶解态与颗粒态. 尽管实际上溶解态与颗粒态的界限并不清晰, 然而这一方法几乎已成为公认的标准方法. 通过过滤方法得到的天然有机质的性质与滤膜的孔径相关. 此 外通过反渗透方法从天然水体中提取天然有机质已被广泛采用, 而这种方法得到的天然有机质并没有专门 的名称. 通常所见的腐殖酸、富里酸等则是基于化学性质如在酸、碱溶液中的溶解情况分类的. 而目前更为 常用的化学分离方法是离子交换树脂法(XAD), 根据不同组分的极性分离. 近些年来离子交换树脂法得到 了快速的发展. 此外还有一些文献按照天然有机质的生物稳定性进行分类. 然而, 采用不同分离方法的结果 就是, 每项研究都是个例. 溶解性有机质的来源及分离方法的不同使得研究的结果差异很大 ${ }^{[57]}$, 导致各项研 究结果之间的相互比较存在困难, 甚至连实验数据的读懂与分析都比较困难. 同时大量的不同的简称加深 了人们对溶解性有机质复杂性的印象,也使研究者望而却步.

\section{3 实验室与野外研究之间的差距}

尽管溶解性有机质的结构目前还不完全清楚,但是已取得了重要进展, 特别是近年来, 人们运用多种先 进技术, 如 ${ }^{13} \mathrm{C} /{ }^{15} \mathrm{~N} /{ }^{31} \mathrm{P}-\mathrm{NMR}$ 、红外光谱、离子亲合色谱、高效液相色谱、高分辨质谱、气体质谱仪和质差热分 析等 ${ }^{[58-60]}$, 对其化学结构有了更多的了解. 其中, 高分辨质谱和核磁共振技术是目前有机质结构特征较好的 研究手段. 现代仪器分析方法可以不破坏有机质原有结构的前提下提供元素组成和官能团信息. 如用体积 排阻色谱、膜透析、超滤等技术对溶解性有机质进行分子量分级. 然而在野外研究中这些先进的表征方法常 常被忽视, 研究者总是选择最简单的方式如 TOC 或 DOC 来表征, 有些甚至没有详细的分离方法介绍, 在讨 
论的时候仅用 DOM 代替, 而忽视了不同的分离处理方法对实验结果带来的差异. 因而众多文献中的 DOM 实际上是相差很大的. 由于天然环境中的溶解性有机质结构复杂, 很多研究者选择人工合成的结构简单的 腐殖质代替物或者国际腐殖酸协会提取的标准物质作为研究对象, 而忽视了解决实际问题的初衷, 导致这 一方向研究的实际意义越来越不明显.

\section{4 总结与展望}

天然水体中广泛存在的溶解性有机质是重要的环境介质, 其对污染物环境行为与生物毒性的影响已是 环境领域的焦点. 然而溶解性有机质并非惰性物质, 它与水生生物间直接的作用影响着生物的生理生化特 性, 能激发生物自身的防御系统, 这种轻度的刺激作用不仅对生物具有重要的生态学意义, 而且对污染物的 生物效应产生影响,其自身的生物效应也应引起我们的关注.

实际环境中污染物的迁移转化及生态效应是当前环境领域内的研究热点, 环境因子对生物自身的生态 学作用将会对污染物的生物吸收与毒性效应产生影响. 溶解性有机质是天然水体中重要的环境因子之一, 深人研究其对生物自身的作用及这些作用在污染物的生物浓缩与致毒过程中产生的影响, 对实际环境中污 染物的生态风险评价有着重要的意义.

然而溶解性有机质自身的复杂性、生物效应的轻微性, 及相关研究中的问题导致溶解性有机质的生物 效应的相关研究进展缓慢.

对溶解性有机质的生物效应研究的出发点应明确为解决实际问题, 建立溶解性有机质分离提取的标准 方法, 在研究中应将小分子模型物质与实际的溶解性有机质样品结合, 利用已有先进的技术对实际样品进 行表征, 对各不同来源的溶解性有机质自身的生物效应进行系统的研究, 明确其生物效应是否显著且广泛 存在及主要的作用分子结构与机制.

在系统了解溶解性有机质自身生物效应及机制的基础上, 进一步研究它与其它环境污染物之间的联合 毒性. 因溶解性有机质不同于一般的环境污染物, 它伴随着生物的进化过程, 需要从生态学角度研究这一与 生物的直接作用对生物的最终利弊.

\section{5 参考文献}

[ 1 ] Tsiridis V, Petala M, Samaras P et al. Interactive toxic effect of heavy metals and humic acids on Vibrio fischeri. Ecotoxicology and Environmental Safety, 2006, 63(1): 158-167.

[2] 吴丰昌,王立英,黎 文等. 天然有机质及其在地表环境中的重要性. 湖泊科学,2008,20(1):1-12.

[ 3 ] Chefetz B, Hadar Y, Chen Y. Dissolved organic carbon fractions formed during composting of municipal solid waste: Properties and significance. Acta Hydrochimica et Hydrobiologica, 1998,26 (3) : 172-179.

[ 4 ] Alberts JJ, Takacs M, Egeberg PK. Total luminescence spectral characteristics of natural organic matter( NOM) size fractions as defined by ultrafiltration and high performance size exclusion chromatography (HPSEC). Organic Geochemistry, $2002,33(7): 817-828$.

[ 5 ] Chin WC, Orellana MV, Verdugo P. Spontaneous assembly of marine dissolved organic matter into polymer gels. Nature, $1998,391(6667)$ : 568-572.

[ 6 ] 吴丰昌,金相灿, 张润宇等. 论有机氮磷在湖泊水环境中的作用和重要性. 湖泊科学,2010,22(1): 1-7.

[ 7 ] Akkanen J, Kukkonen JVK. Measuring the bioavailability of two hydrophobic organic compounds in the presence of dissolved organic matter. Environmental Toxicology and Chemistry, 2003, 22(3) : 518-524.

[ 8 ] Wu FC, Tanoue E. Isolation and partial characterization of dissolved copper-complexing ligands in streamwaters. Environmental Science \& Technology, 2001, 35(18) : 3646-3652.

[9] 苒 勇, 孙 可, 马晓轩等. 土壤和沉积物中的聚合有机质对多环芳烃分布和提取的影响. 生态毒理学报, 2006, $1(4)$ : 336-342.

[10] Smith DS, Wu FC. Preface: Metal interactions with natural organic matter. Applied Geochemistry, 2007, $22(8): 1567$.

[11] Bai Y, Wu FC, Liu C et al. Interaction between carbamazepine and humic substances: A fluorescence spectroscopy study. Environmental Toxicology and Chemistry, 2008, 27(1): 95-102.

[12] Haitzer M, Hoss S, Traunspurger W et al. Effects of dissolved organic matter( DOM) on the bioconcentration of organic chemicals in aquatic organisms. Chemosphere, 1998, 37(7): 1335-1362.

[13] Suffet IH, Williams LL, Jafvert CT et al. Synopsis of discussion session: Influences of particulate and dissolved material 
on the bioavailability of organic compounds. In: Hamelink JL, Landrum PF, Bergman HL et al eds. Bioavailability: Physical, chemical and biological interactions. Boca Raton: CRC Press, 1994: 93-108.

[14] De Paolis F, Kukkonen J. Binding of organic pollutants to humic and fulvic acids: Influence of $\mathrm{pH}$ and the structure of humic material. Chemosphere, 1997, 34(8) : 1693-1704.

[15] Ke RH, Luo JP, Sun LW et al. Predicting bioavailability and accumulation of organochlorine pesticides by Japanese meda$\mathrm{ka}$ in the presence of humic acid and natural organic matter using passive sampling membranes. Environmental Science \& Technology, 2007, 41(19): 6698-6703.

[16] Qiao P, Farrell AP. Influence of dissolved humic acid on hydrophobic chemical uptake in juvenile rainbow trout. Comparative Biochemistry and Physiology C-Toxicology and Pharmacology, 2002, 133(4) : 575-585.

[17] Chen S, Ke RH, Huang SB et al. Impact of dissolved humic acid on the bioavailability of acenaphthene and chrysene assessed by membrane-based passive samplers. Chinese Science Bulletin, 2007, 52 (19) : 2642-2648.

[18] Haitzer M, Hoss S, Traunspurger W et al. Effects of quantity, quality, and contact time of dissolved organic matter on bioconcentration of benzo[a] pyrene in the nematode Caenorhabditis elegans. Environmental Toxicology and Chemistry, $1999, \mathbf{1 8}(3): 459-465$.

[19] Steinberg CEW, Paul A, Pflugmacher S et al. Pure humic substances have the potential to act as xenobiotic chemicals-A review. Fresenius Environmental Bulletin, 2003, 12(5) : 391-401.

[20] Steinberg CEW, Kamara S, Prokhotskaya VY et al. Dissolved humic substances-ecological driving forces from the individual to the ecosystem level? Freshwater Biology, 2006, 51(7): 1189-1210.

[21] Munster U. Investigation about structure, distribution and dynamics of different organic substrates in the DOM of lake Plusssee. Archiv fiur Hydrobiologie Supplement, 1985 ,70 : 429-480.

[22] Visser SA. Physiological action of humic substances on microbial cells. Soil Biology \& Biochemistry, 1985, 17 (4) : 457462.

[23] Maurice PA, Manecki M, Fein JB et al. Fractionation of an aquatic fulvic acid upon adsorption to the bacterium, Bacillus subtilis. Geomicrobiology Journal, 2004, $21(2)$ : 69-78.

[24] Elayan NM, Treleaven WD, Cook RL. Monitoring the effect of three humic acids on a model membrane system using P-31 NMR. Environmental Science \& Technology, 2008, 42(5) : 1531-1536.

[25] Vigneault B, Percot A, Lafleur M et al. Permeability changes in model and phytoplankton membranes in the presence of aquatic humic substances. Environmental Science \& Technology, 2000, 34 (18) : 3907-3913.

[26] Glover CN, PaneEF, Wood CM. Humic substances influence sodium metabolism in the freshwater crustacean Daphnia magna. Physiological and Biochemical Zoology, 2005, 78(3): 405-416.

[27] Manusadzianas L, Maksimov G, Darginaviciene J et al. Response of the charophyte Nitellopsis obtusato heavy metals at the cellular, cell membrane, and enzyme levels. Environmental Toxicology, 2002, 17: 275-283.

[28] Galvez F, Donini A, Playle RC et al. A matter of potential concern: natural organic matter alters the electrical properties of fish gills. Environmental Science \& Technology, 2008, 42(24) : 9385-9390.

[29] Cazenave J, Bistoni M, Zwirnmann E et al. Attenuating effects of natural organic matter on microcystin toxicity in zebra fish (Danio rerio) embryos-Benefits and costs of microcystin detoxication. Environmental Toxicology, 2006, 21(1) : 22-32.

[30] Menzel R, Sturzenbaum S, Barenwaldt A et al. Humic material induces behavioral and global transcriptional responses in the nematode Caenorhabditis elegans. Environmental Science \& Technology, 2005, 39(21) : 8324-8332.

[31] Matsuo AYO, Woodin BR, Reddy CM et al. Humic substances and crude oil induce cytochrome P450 $1 \mathrm{~A}$ expression in the Amazonian fish species Colossoma macropomum (Tambaqui). Environmental Science \& Technology, 2006, 40 (8): 2851-2858.

[32] Timofeyev MA, Shatilina ZM, Bedulina DS et al. Natural organic matter(NOM) has the potential to modify the multixenobiotic resistance(MXR) activity in freshwater amphipods Eulimnogammarus cyaneus and E. verrucosus. Comparative Biochemistry and Physiology B-Biochemistry \& Molecular Biology, 2007, 146(4) : 496-503.

[33] Hoss S, Bergtold M, Haitzer M et al. Refractory dissolved organic matter can influence the reproduction of Caenorhabditis elegans( Nematoda). Freshwater Biology, 2001, 46(1): 1-10.

[34] Steinberg CEW, Hoss S, Bruggemann R. Further evidence that humic substances have the potential to modulate the fertilityof the nematode Caenorhabditis elegans. Intern Rev Hydrobiol, 2002, 87 (1) : 121-133.

[35] Meinelt T, Schreckenbach K, Knopf $\mathrm{K}$ et al. Humic substances affect physiological condition and sex ratio of swordtail (Xiphophorus helleri Heckel). Aquatic Sciences, 2004, 66(2) : 239-245.

[36] Lutz I, Jie Z, Opitz R et al. Environmental signals: Synthetic humic substances act as xeno-estrogen and affect the thyroid system of Xenopus laevis. Chemosphere, 2005, 61(8) : 1183-1188.

[37] Steinberg CEW, Meinelt T, Timofeyev MA et al. Humic substances. Environmental Science and Pollution Research, $2008, \mathbf{1 5}(2): 128-135$. 
[38] Campbell PGC, Twiss MR, Wilkinson KJ. Accumulation of natural organic matter on the surfaces of living cells: implications for the interaction of toxic solutes with aquatic biota. Canadian Journal of Fisheries and Aquatic Sciences, 1997, 54 (11) : 2543-2554.

[39] Manusadzianas L, Maksimov G, Darginaviciene J et al. Response of the charophyte Nitellopsis obtusa to heavy metals at the cellular, cell membrane, and enzyme levels. Canada: 10th International Symposium on Toxicity Assessment, 2001 : 275-283.

[40 ] Timofeyev MA, Shatilina ZM, Kolesnichenko AV et al. Specific antioxidant reactions to oxidative stress promoted by natural organic matter in two amphipod species from Lake Baikal. Environmental Toxicology, 2006, 21(2) : 104-110.

[41] Timofeyev MA, Shatilina ZM, Kolesnichenko AV et al. Natural organic matter( NOM) induces oxidative stress in freshwater amphipods Gammarus lacustris Sars and Gammarus tigrinus(Sexton). Science of the Total Environment, 2006, 366(2$3)$ : 673-681.

[42] Cooper WT, Stenson A, Milligan L et al. Ultrahigh resolution mass spectrometry of aquatic humic substances: recurring molecular themes and polymeric character. In: Martin Neto L, Milori DMBP, da Silva WTL eds. Humic substances and soil and water environment. Brazil: Embrapa, 2004: 257-260.

[43 ] Hatcher P, Kim S, Sugiyama Y. Intercomparisons of some new approaches for investigating the molecular weight distribution of dissolved organic matter. In: Martin Neto L, Milori DMBP, da Silva WTL eds. Humic substances and soil and water environment. Brazil: Embrapa, 2004 : 241-243.

[44] Timofeyev MA, Wiegand C, Burnison BK et al. Impact of natural organic matter( NOM) on freshwater amphipods. Science of the Total Environment, 2004, 319(1-3) : 115-121.

[45] Wiegand C, Meems N, Timoveyev M et al. More evidence for humic substances acting as biogeochemicals on organisms. In: Ghabbour EA, Davies G eds. Humic substances: Nature's most versatile materials. New York: Taylor and Francis, 2004 : 349-362.

[46] Bittner M, Janosek J, Hilscherova K et al. Activation of Ah receptor by pure humic acids. Environmental Toxicology, 2006, 21 (4) : 338-342.

[47] Bittner M, Hilscherova K, Giesy JP. Changes of AhR-mediated activity of humic substances after irradiation. Environment International, 2007, 33(6) : 812-816.

[48 ] Laurberg P, Andersen S, Pedersen IB et al. Humic substances in drinking water and the epidemiology of thyroiddisease. Biofactors, 2003, 19(3-4) : 145-153.

[49] Janosek J, Bittner M, Hilscherova K et al. AhR-mediated and antiestrogenic activity of humic substances. Chemosphere, 2007, $67(6)$ : 1096-1101.

[50 ] Ter Laak TL, Van Eijkeren JCH, Busser FJM et al. Facilitated transport of polychlorinated biphenyls and polybrominated diphenyl ethers by dissolved organic matter. Environmental Science \& Technology, 2009, 43(23) : 1379-1385.

[51] Slaveykova VI. Predicting Pb bioavailability to freshwater microalgae in the presence of fulvic acid: Algal cell density as a variable. Chemosphere, 2007,69(9): 1438-1445.

[52] Borrok D, Aumend K, Fein JB. Significance of ternary bacteria-metal-natural organic matter complexes determined through experimentation and chemical equilibrium modeling. Chemical Geology, 2007, 238(1-2) : 44-62.

[53] Lamelas C, Pinheiro JP, Slaveykova VI. Effect of humic acid on $\mathrm{Cd}(\mathrm{II}), \mathrm{Cu}$ ( II ), and $\mathrm{Pb}$ ( II ) uptake by freshwater algae: kinetic and cell wall speciation considerations. Environmental Science \& Technology, 2009, 43(3) : 730-735.

[54] Steinberg CEW, Saul N, Pietsch K et al. Dissolved humic substances facilitate fish life in extreme aquatic environments and have the potential to extend the lifespan of Caenorhabditis elegans. Annals of Environmental Science, 2007, 1: 81-90.

[55] Wiegand C, Pehkonen S, Akkanen J et al. Bioaccumulation of paraquat by Lumbriculus variegatus in the presence of dissolved natural organic matter and impact on energy costs, biotransformation and antioxidative enzymes. Chemosphere, $2007, \mathbf{6 6}(3)$ : $558-566$.

[56] Filella M. Freshwaters: which NOM matters? Environmental Chemistry Letters, 2009,7 : 21-35.

[57] Sharp JH. Analytical methods for total DOM pools. In: Hansell DA, Carlson CA eds. Biogeochemistry of marine dissolved organic matter. Amsterdam: Academic Press, 2002: 35-58.

[58 ] Wu FC, Evans RD, Dillon PJ. High-performance chromatographic fractionation and characterization of fulvic acid. Analytica Chimica Acta, 2002, 464(1): 47-55.

[59] Wu FC, Evans RD, Dillon PJ. Separation and characterization of NOM by high-performance liquid chromatography and on-line three-dimensional excitation emission matrix fluorescence detection. Environmental Science \& Technology, 2003, $37(16)$ : 3687-3693.

[60] Fu PQ, Liu CQ, Wu FC. Three-dimensional excitation emission matrix fluorescence spectroscopic characterization of dissolve organic matter. Spectroscopy and Spectral Analysis, 2005, 25 (12) : 2024-2028. 